\title{
Evaluation of Supervised Classification Algorithms for Human Activity Recognition with Inertial Sensors
}

\author{
Tahmina Zebin \\ School of EEE \\ University of Manchester, UK \\ tahmina.zebin@manchester.ac.uk
}

\author{
Patricia J. Scully \\ School of CEAS \\ University of Manchester, UK \\ patricia.scully@manchester.ac.uk
}

\author{
Krikor B. Ozanyan \\ School of EEE \\ University of Manchester, UK \\ k.ozanyan@manchester.ac.uk
}

\begin{abstract}
The main aim of this work is to compare the performance of different algorithms for human activity recognition by extracting various statistical time domain and frequency domain features from the inertial sensor data. Our results show that Support Vector Machines with quadratic kernel classifier (accuracy: 93.5\%) and Ensemble classifier with bagging and boosting (accuracy: 94.6\%) outperforms other known activity classification algorithms. A parallel coordinate plot based on visualization of features is used to identify useful features or predictors for separating classes. This enabled exclusion of features that contribute least to classification accuracy in a multi-sensor system (five in our case), made the classifier lightweight in terms of number of useful features, training time and computational load and lends itself to real-time implementation.
\end{abstract}

Keywords- Human Activity Recognition (HAR), Classification, Feature Selection, Supervised Machine Learning, $M A T L A B$

\section{INTRODUCTION}

The demands for understanding human activities have grown enormously in recent years for ubiquitous computing, human computer interaction, and domains such as elder care support, rehabilitation assistance, and cognitive disorder recognition systems $[1,2]$. Despite significant research efforts over the past few decades, activity recognition still remains a challenging problem. Wearable sensor based Human Activity Recognition (HAR) described in literature uses sensors such as accelerometer, gyroscope and pressure sensors to monitor and recognize activities $[2,3]$. The information obtained from human physical activity is valuable in the long-term assessment of biomechanical parameters and physiological variables. Typically, the goal of these studies is to develop methods to predict the activity or activities which may in turn be used for determining normal or abnormal patterns, which can then be used to support care of the elderly, the chronically ill and people with special needs [4].

In this paper, we aim to investigate advanced signal processing and machine learning techniques to improve classification accuracy and prediction speed of daily life activities from inertial sensor(accelerometric and gyroscopic) data. We used supervised classification methods for recognizing six daily-life activities using five MPU-9150 inertial sensors placed on the lower body of a human subject. Features were extracted from the raw acceleration and gyroscopic data collected from these sensors, and then algorithms such as Decision Tree, Linear and Quadratic Discriminant Analysis, Support Vector Machines, Ensemble classifier with bagging and boosting used for activity recognition. We obtained classification accuracy up to $94.3 \%$ for the activity recognition scenario, which outperformed the results reported in the relevant literature.

\section{RELATED WORK AND BACKGROUND}

Some early activity recognition studies are found to be carried out in early 2000's by Laerhoven et al. [5] and Aminian et al. [6]. Also, Bao \& Intile [7] classification with accelerometer data for 20 activities. In their experiments they used 5 biaxial accelerometers on different parts of the body and then collected data from activities like walking, sitting, standing still, watching TV, running, stretching, scrubbing, folding laundry, climbing Stairs, etc. They used the data collected to train the classifiers $\mathrm{C} 4.5$ decision tree, decision table, k-nearest neighbor (K-NN) and Naive Bayes (NB) available as algorithms in the WEKA[8] toolbox. The classifiers were tested with the following features: standard deviation, energy distribution, DC component, Entropy, and correlation coefficients. The best overall accuracy of $84 \%$ was obtained with the C4.5 classifiers. Ravi et al. conducted the recognition of activities using a number of classifiers by processing the data collected from a single subject in multiple settings $[9,10]$ They concluded that activities like standing, walking, running, going up and downstairs, can be recognized with accuracy up to $90.61 \%$ using a single 3-axis accelerometer. Manini and Sabatini implemented and tested Hidden Markov Models and Gaussian Mixture Models based classifiers for activity recognition [11], but these are computationally complex classifiers. Despite significant research efforts over the past few decades, activity recognition still remains a challenging problem because of the need to design highly accurate yet lightweight classifiers.

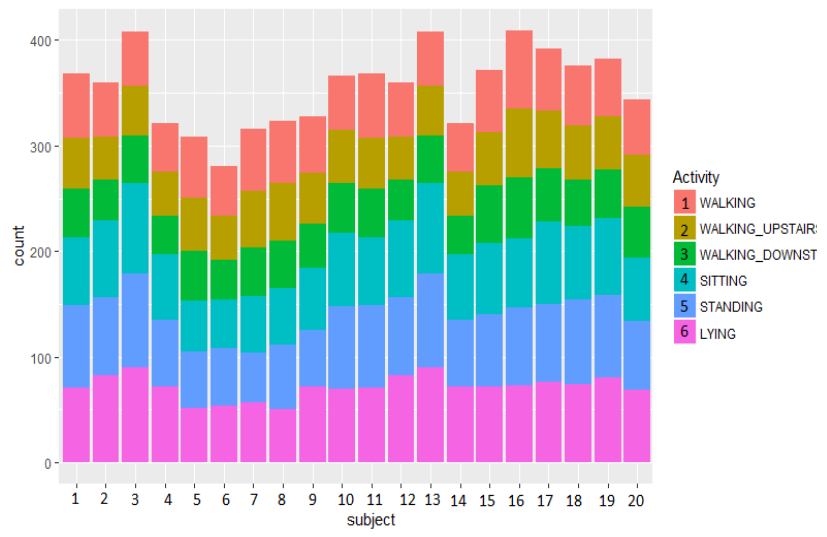

Fig. 1. Subject wise (volunteer) distribution of Activity types and Sensor Data segments in the collected dataset. 
For this research, we collected data from 20 healthy volunteers (age[y]: 24.6 \pm 6.9 ; height [m]: $1.63 \pm 0.6$, weight [kg]: $64.7 \pm 7.1$ ) while undertaking six common daily life activities such as, (1)walking, (2)walking upstairs, (3) downstairs, (4)sitting, (5)standing (6) lying down. Figure 1 shows the distribution of data segments which are fairly evenly distributed across different volunteers and activity types they conducted.

We calculated features or predictors such as average and root mean squared amplitude, value of the Autocorrelation coefficients and position of the peaks, spectral peak and amplitude from inertial sensor activity data. We used the Classification Learner app[12] in MATLAB 2016b to explore supervised machine learning approach using various classifiers. Sixty Six handcrafted features [4] were used as predictors for the classifiers, and 5-fold cross validation scheme(to avoid overfitting) was utilized. Models were trained and we have assessed the results with confusion matrices under the same experimental situations. Aautomated training was performed to search for the best classification model type, including decision trees, discriminant analysis, support vector machines, logistic regression, nearest neighbours, and ensemble classification. All computation tasks were performed on a Lenovo Yoga laptop with an Intel Core i7 processor (CPU @2.5GHz, 2-cores, 4logical Processors) and 8GB physical memory while training these models.

\section{EXPERIMENTAL RESULTS AND DISCUSSION}

From the results listed in Table I, it is seen that the classification accuracy changes when parameters, such as number of splits, type of kernel, number of neighbors or learners, are modified for different algorithms. An improvement (3.9\%) in recognition accuracy was seen for a Decision tree classifier when the number of splits increases from 20 to 100 following Gini's Diversity Index as the split criterion[13]. Linear and Quadratic Discriminant analysis was not found to be suitable for classifying activity from the inertial sensor data because the covariance of the predictors did not produce sufficient discrimination between classes. SVM classifier with quadratic kernel, adapted for a multi-class activity classification scenario, achieves an accuracy of $93.5 \%$ and Ensemble classifier with bagging [14] and boosting obtains $94.3 \%$ accuracy, which outperforms all other known activity classification algorithms.

However, if the algorithm has to be implemented for a realtime application, the overall training time and prediction speed has to be reasonably fast. Though Ensemble classifier had recognition accuracy higher than Quadratic SVM, SVM will perform better in real-time scenario because of its faster processing time and prediction speed. Figure 2 presents the confusion matrix for the two best performing models in terms of accuracy and prediction speed.

TABLE I. QUANTITATIVE PERFORMANCE COMPARISON OF THE CLASSIFIERS

\begin{tabular}{|c|c|c|c|c|}
\hline Classifier & Further information & $\begin{array}{c}\text { Average Accuracy over 5-Fold } \\
\text { Cross-Validation (\%) }\end{array}$ & $\begin{array}{c}\text { Training } \\
\text { time(s) }\end{array}$ & $\begin{array}{c}\text { Prediction } \\
\text { Speed(observations/sec) }\end{array}$ \\
\hline Complex Decision tree & $\begin{array}{l}\text { No. of Splits: } 100 \\
\text { Split Criterion: Gini’s Diversity Index }\end{array}$ & $91.8 \%$ & 7.6294 & $\sim 8000$ \\
\hline Medium Decision Tree & $\begin{array}{l}\text { No. of Splits: } 20 \\
\text { Split Criterion: Gini's Diversity Index }\end{array}$ & $87.9 \%$ & 3.8345 & $\sim 7000$ \\
\hline Linear Discriminant & Regularization: Diagonal Covariance & $80.2 \%$ & 1.2572 & $\sim 9300$ \\
\hline Quadratic Discriminant & Regularization: Diagonal Covariance & $72.3 \%$ & 3.2572 & $\sim 900$ \\
\hline SVM & $\begin{array}{l}\text { Multiclass Method: one-vs-one, } \\
\text { Standardization: true }\end{array}$ & $\begin{array}{l}\text { 91.8\%(Linear kernel) } \\
\text { 93.5\%(Quadratic kernel) } \\
\text { 93.0\%(Cubic kernel) }\end{array}$ & $\begin{array}{l}4.192 \\
4.947 \\
7.275 \\
\end{array}$ & $\sim 8500$ \\
\hline KNN & Distance matrix Euclidean & $87.0 \%(\mathrm{n}=10)$ & 16.517 & $\sim 910$ \\
\hline Ensemble & $\begin{array}{l}\text { No. of learners: } 30 \\
\text { Subspace dimension:33 }\end{array}$ & $\begin{array}{l}\text { 94.6\%( Bagged) } \\
90.3 \%(\text { Subspace KNN) }\end{array}$ & $\begin{array}{l}64.975 \\
64.90\end{array}$ & $\begin{array}{l}\sim 1700 \\
\sim 83\end{array}$ \\
\hline
\end{tabular}

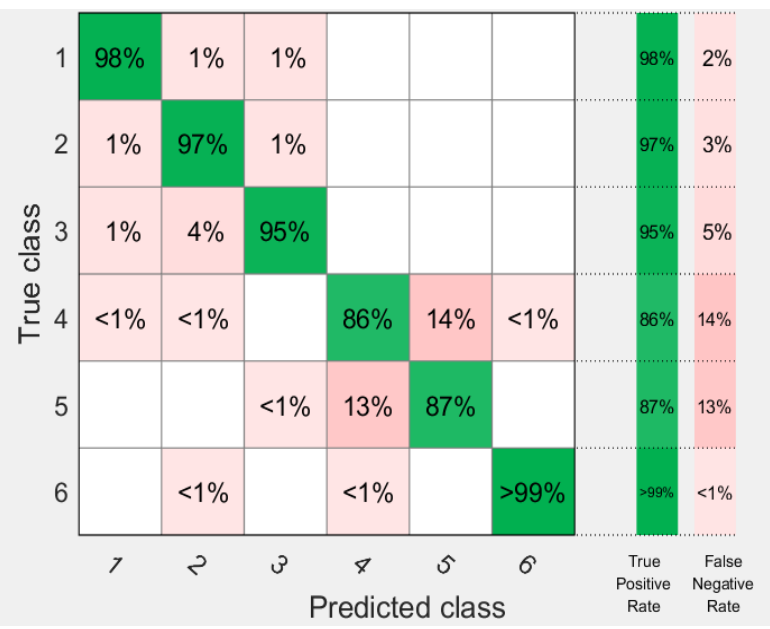

b)

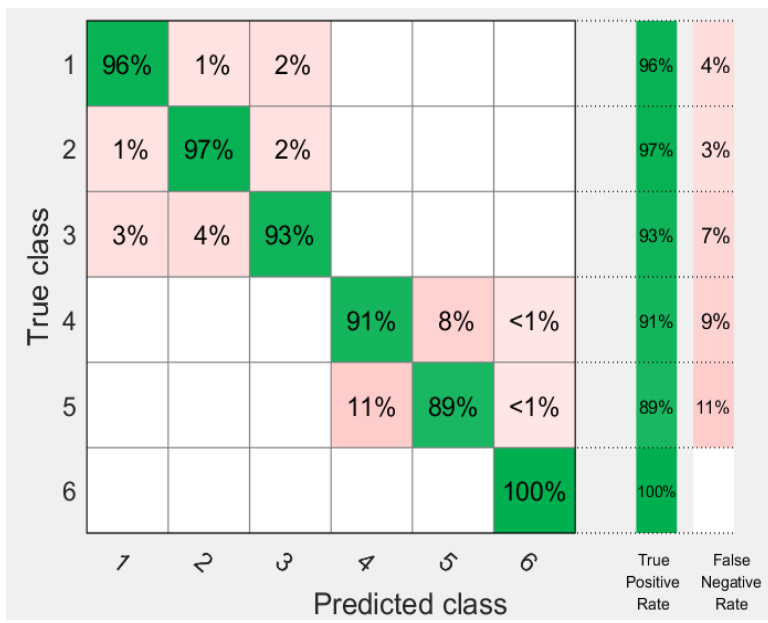

Fig. 2. Confusion Matrix for a) Quadratic SVM; and b) Ensemble (bagged Decision Tree) Classifier for six common daily life activity classes (1-walking, 2-walking upstairs, 3- downstairs, 4-sitting, 5-standing, 6-lying down) 


\section{IMPROVING THE CLASSIFIER: FEATURE SELECTION WITH THE PARALLEL COORDINATES PLOT AND OTHER TECHNIQUES}

Here we note, that the number of time and frequency domain features (predicting variables) obtained from the accelerometer and gyroscope affects the performance of the classifier differently. It was observed that the gyroscope data contained far less useful features than the accelerometer data. However, features from a gyroscope improve the accuracy in the case where the activities are constrained and distinguished by translation and rotation of the joint angles. For example, sensors that are placed on thighs and shanks have different orientation during activities like sitting and standing, or when discriminating cases of level walking, walking upstairs or downstairs. During this research, to further reduce the computational load of the classifier, we employed feature selection techniques to discard the features not contributing much to the response of the classification algorithm. To investigate which features to include or exclude, the parallel coordinates plot (Fig.3) was used to find the relationships between features and identify useful predictors for separating classes. The training data and misclassified points (shown in dashed lines) can be visualized on the parallel coordinates plot. These features can also be selected sequentially or by using Correlation Feature Selection Method based filtering [15] or by using predictors transformed by principal component analysis to design accurate yet lightweight classifiers.

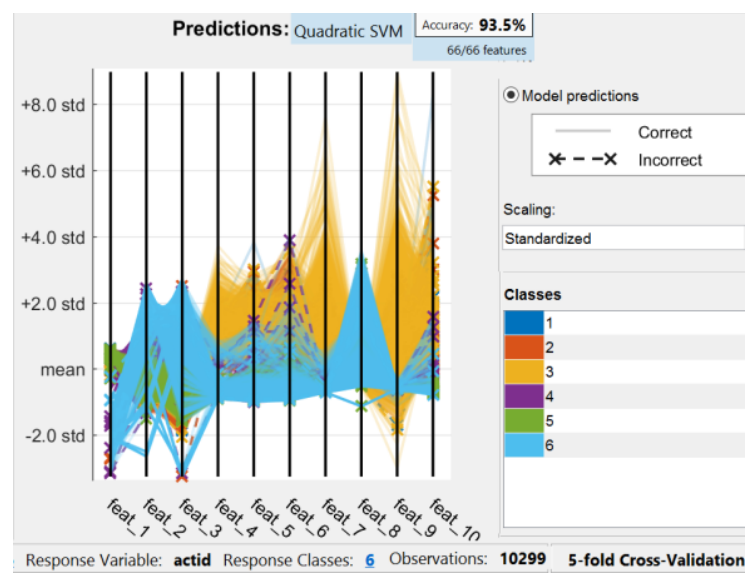

Fig. 3. Parallel Coordinate Plot to observe the impact of features on classification accuracy. For example, feat_1, feat_6 and feat_10 produces many misclassified points, hence are not ideal for prediction purposes in our classification scenario, misclassified points are shown as dashed lines on the plot. The mean of each predictor is standardized at zero and scaled the predictors by their standard deviations. (The detailed feature map for this can be found in ref [4])

\section{CONCLUSION}

The presented work investigated an inertial sensor based human activity recognition system and its efficacy in a multi-class activity recognition scenario. We tailored algorithms in MATLAB to classify the dataset we collected and hence it provided generalization in the procedural stages involved in this research and will contribute in inferring some intuitive decisions for human activity recognition based research. Further research will include composite activities such as running and jogging, concurrent and overlapping activities and also some multi-attribute classification approaches [3]. Therefore, we plan to verify the effectiveness of the models by testing it on other challenging activities such as cycling, typing, bicep curl, dead lift, bench press, etc.

\section{REFERENCES}

[1] N. Twomey, T. Diethe, I. Craddock, and P. Flach, "Unsupervised learning of sensor topologies for improving activity recognition in smart environments," Neurocomputing, vol. 234, pp. 93-106, 2017.

[2] G. M. Jeong, P. H. Truong, and S. I. Choi, "Classification of Three Types of Walking Activities Regarding Stairs Using Plantar Pressure Sensors," IEEE Sensors Journal, vol. 17, pp. 2638-2639, 2017.

[3] O. D. Lara and M. A. Labrador, "A Survey on Human Activity Recognition using Wearable Sensors," IEEE Communications Surveys \& Tutorials, vol. 15, pp. 1192-1209, 2013.

[4] T. Zebin, P. J. Scully, and K. B. Ozanyan, "Inertial Sensor Based Modelling of Human Activity Classes: Feature Extraction and Multi-sensor Data Fusion Using Machine Learning Algorithms," in eHealth $360^{\circ}$ : International Summit on eHealth, Budapest, Hungary, June 14-16, 2016, Revised Selected Papers, K. Giokas, L. Bokor, and F. Hopfgartner, Eds., ed Cham: Springer International Publishing, 2017, pp. 306-314.

[5] K. V. Laerhoven, K. A. Aidoo, and S. Lowette, "Real-time analysis of data from many sensors with neural networks," in Proceedings Fifth International Symposium on Wearable Computers, 2001, pp. 115-122.

[6] K. Aminian, B. Najafi, C. Büla, P. F. Leyvraz, and P. Robert, "Spatio-temporal parameters of gait measured by an ambulatory system using miniature gyroscopes," Journal of Biomechanics, vol. 35, pp. 689-699, 2002.

[7] L. Bao and S. S. Intille, "Activity Recognition from UserAnnotated Acceleration Data," in Pervasive Computing: Second International Conference, PERVASIVE 2004, Linz/Vienna, Austria, April 21-23, 2004. Proceedings, A. Ferscha and F. Mattern, Eds., ed Berlin, Heidelberg: Springer Berlin Heidelberg, 2004, pp. 1-17.

[8] M. Hall, E. Frank, G. Holmes, B. Pfahringer, P. Reutemann, and I. H. Witten, "The WEKA data mining software: an update," SIGKDD Explor. Newsl., vol. 11, pp. 10-18, 2009.

[9] N. Ravi, N. Dandekar, P. Mysore, and M. L. Littman, "Activity recognition from accelerometer data," presented at the Proceedings of the 17th conference on Innovative applications of artificial intelligence - Volume 3, Pittsburgh, Pennsylvania, 2005.

[10] D. Ravi, C. Wong, B. Lo, G. Z. Yang, and Ieee, "Deep Learning for Human Activity Recognition: A Resource Efficient Implementation on Low-Power Devices," in 2016 Ieee 13th International Conference on Wearable and Implantable Body Sensor Networks, ed, 2016, pp. 71-76.

[11] A. Mannini and A. M. Sabatini, "Machine Learning Methods for Classifying Human Physical Activity from On-Body Accelerometers," Sensors, vol. 10, p. 1154, 2010.

[12] Classification Learner app for Supervised Machine Learning. Available:https://uk.mathworks.com/help/stats/classificationlear ner-app.html

[13] M. Kantardzic, "Decision Trees and Decision Rules," in Data Mining, ed: John Wiley \& Sons, Inc., 2011, pp. 169-198.

[14] L. Breiman, "Bagging Predictors," Machine Learning, vol. 24, pp. 123-140, 1996

[15] K. Michalak and H. Kwasnicka, "Correlation based feature selection method," Int. J. Bio-Inspired Comput., vol. 2, pp. 319332,2010 